\title{
Editorial: Bioactive Substances from Medicinal Plants for Metabolic Disorders
}

Article in Current Medicinal Chemistry · March 2017

DOI: $10.2174 / 092986732404170302223350$

CITATIONS

0

4 authors:

\section{Tânia R. Dias}

Universidade da Beira Interior

47 PUBLICATIONS 384 CITATIONS

SEE PROFILE

Pedro F Oliveira

University of Porto

210 PUBLICATIONS 1,881 CITATIONS

SEE PROFILE
READS

58
Marco G. Alves

Universidade da Beira Interior

201 PUBLICATIONS 1,805 CITATIONS

SEE PROFILE

Branca Maria Silva

Universidade da Beira Interior

131 PUBLICATIONS 2,717 CITATIONS

SEE PROFILE

Some of the authors of this publication are also working on these related projects:

Project

Project
Obesitility - Gut hormones and testicular metabolism: mechanisms associated with obesity-related male infertility View project

Aquaporins as molecular partners of CFTR in the Blood-Testis Barrier: a crossroad between human male infertility and cystic fibrosis? View project

All content following this page was uploaded by Tânia R. Dias on 11 March 2017.

The user has requested enhancement of the downloaded file. 


\section{Editorial}

\section{Bioactive Substances from Medicinal Plants for Metabolic Disorders}

Metabolic disorders, particularly Diabetes Mellitus (DM), are major causes of death worldwide. In fact, the world statistics for DM are alarming: this epidemic disease kills one person every six seconds, being the fourth cause of death. Besides the health issues, the costs associated with DM, and its co-morbidities, are massive and the pressure in National Health Care Systems is dramatically increasing. Patients with DM, particularly those with type 2 diabetes (T2D), are primarily advised to change their dietary habits and exercise regime. However, as the disease progresses and becomes more severe, conventional drugs are prescribed. Most of these patients need polypharmacological therapy and though progresses have been made, efforts are mandatory in a search for a "perfect" antidiabetic drug.

In this context, plant-derived therapies have been used since ancient times for the treatment of several diseases, including metabolic disorders, such as DM. Indeed, there are several plant-derived products in the pharmaceutical industry, thus illustrating the potential of plants as source of compounds that may be useful in human health. Literature has shown that several plants and phytochemicals present promising properties against the health problems promoted by metabolic diseases. This field has emerged as a hot topic of research and there are a few compounds under investigation that may come to the market in the next years. Thus, in this special issue of Current Medicinal Chemistry, the chemistry of bioactive substances from medicinal plants and their possible contribution to the search of new, cost-effective and safe agents to prevent or treat metabolic disorders, especially DM and its associated comorbidities, are highlighted. The effects of plant-derived substances in metabolic disorders-associated dysfunctions (neurodegeneration, cardiovascular diseases, infertility, aging, hyperglycemia, among others) are also discussed, as well as the future perspectives for their medicinal use.

Phenolic compounds constitute one of the most abundant and bioactive group of phytochemicals in edible and medicinal plants, presenting antioxidant, anti-inflammatory and antidiabetic properties, between many others. Prof. Silva's group describes the most important classes of phenolic compounds and discusses how dietary (poly)phenols can have protective effects against DM-associated co-morbidities.

Quercetin is a flavonoid widely distributed in plants and with a significant presence in human diet. It has several important properties, including a well-known antioxidant and anti-inflammatory effect. Prof. Haddad' group presents a review focused on the role of this polyphenol in the prevention and treatment of several metabolicassociated disorders, such as diabetes, cancer, neurodegenerative or cardiovascular diseases.

Daidzein is a soy isoflavone that has been reported to have biological effects in several tissues due to its high penetrating capacity. It also presents both estrogenic and antiestrogenic effects. Prof. Nabavi's group discusses the phytomedicinal potential of this polyphenol, especially on brain, since it is particularly sensitive to oxidative stress induced by hyperglycemia, which eventually may lead to neurodegeneration.

Coffee is one of the most consumed drinks in the world. After decades of "bad reputation", there is an increasing amount of literature focused on its health benefits, namely against metabolic disorders, such as DM. Prof. Casal's group discusses if the consumption of coffee-based beverages can be used as a dietary intervention to counteract some of the deleterious effects of T2D. 
Glycogen phosphorylase has also emerged as a molecular target for the discovery of new hypoglycemic agents. Several bioactive substances from medicinal plants show an ability to modulate glycogen phosphorylase activity. Prof. Leonidas's group focused their attention on glycogen phosphorylase inhibitors, particularly flavonoids, tannins and pentacyclic triterpenes.

DM is very common during pregnancy and may have catastrophic consequences to the fetal and maternal health. So, this issue has arisen great attention in the last few years. Prof. Volpato's group discuss the possible use of bioactive substances from medicinal plants as an alternative or coadjuvant therapy against DM, especially during pregnancy.

Metabolic disorders, namely DM, frequently cause psychiatric and neurodegenerative problems. Prof. Aliev's group discuss the therapeutic potential of several botanical species from the American and Asian Continents that are used by traditional medicine in the treatment of anxiety.

In summary, this special issue of Current Medicinal Chemistry is focused on the medicinal chemistry and use of bioactive substances from plants to counteract or treat the deleterious effects of metabolic disorders, which remain as one of the major health challenges of modern societies.

Tânia R. Dias ${ }^{1,2}$, Marco G. Alves ${ }^{1}$, Pedro F. Oliveira ${ }^{2,3}$ and Branca M. Silva ${ }^{1}$

Guest Editors

${ }^{1}$ Health Sciences Research Centre University of Beira Interior (CICS-UBI), Covilhã

Portugal

E-mails: taniairdias@gmail.com; bmcms@ubi.pt; alvesmarc@gmail.com

${ }^{2}$ Department of Microscopy Laboratory of Cell Biology Institute of Biomedical Sciences Abel Salazar (ICBAS) and Unit for Multidisciplinary Research in Biomedicine (UMIB) University of Porto, Porto Portugal

E-mail: taniairdias@gmail.com

${ }^{3}$ i3S - Instituto de Investigação e Inovação em Saúde Universidade do Porto, Porto Portugal E-mail: pfobox@gmail.com 